\title{
Determination of the Equilibrium Constant for the Tautomeric 9-Hydroxyanthracene/9-Anthrone System in Aprotic Solvents by a Novel Application of Cyclic Voltammetry
}

\author{
Kristoffer Almdal, Hanne Eggert and Ole Hammerich*
}

Department of General and Organic Chemistry, The H. C. Ørsted Institute, University of Copenhagen, Universitetsparken 5, DK-2100 Copenhagen Ø, Denmark

The voltammetric reduction of benzophenone and related simple aromatic ketones in aprotic media is characterized by the reversible formation of the corresponding anion radicals even at low sweep rates.' However, special structural features, such as for example a hydrogen atom in the $\alpha$-position of the carbonyl group, may cause the ketone to be sufficiently acidic to protonate its own anion radical..$^{2,3}$ As a consequence of this self-protonation reaction, the voltammetric wave may appear as irreversible and the apparent number of electrons transferred, $n$, will be less than one. The exact value of $n$ depends on the stoichiometry of the over-all reaction. ${ }^{4} \mathrm{~A}$ recent voltammetric study of the reduction of 9-anthrone in $N, N$-dimethylformamide (DMF) revealed $^{5,6}$ that this compound belongs to the class of acidic ketones described above (eqn. 1, n= $2 / 3$ ), but with the additional mechanistic complication that the equilibrium between 9-anthrone and the tautomeric form, 9-hydroxyanthracene (eqn. 2), participates in determining the kinetics of the proton transfer process. The value of the equilibrium constant, $K_{2}$, the knowledge of which is pertinent for the detailed analysis of the voltammetric behavior of 9-anthrone, has been determined in DMF and other organic solvents by ${ }^{1} \mathrm{H} \mathrm{NMR}^{7}$ and UV/Vis ${ }^{8}$ spectroscopy. However, in DMF the two methods have resulted in $K_{2}$ values which differ by more than a factor of ten, $0.08^{7}$ and $1.3^{8}$, respectively.

The aim of this communication is to show how $K_{2}$ may be determined in aprotic solvents like

*To whom correspondence should be addressed.

230 Acta Chemica Scandinavica B 40 (1986) 230-232

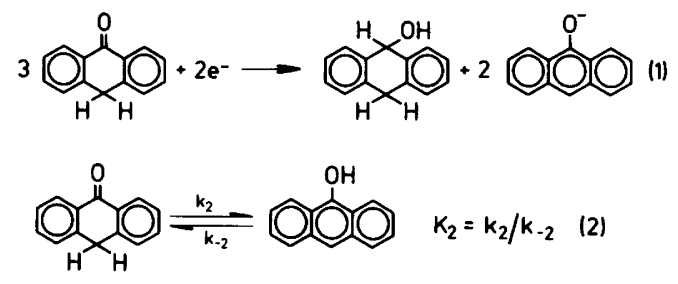

DMF and dimethylsulfoxide (DMSO) by a novel application of cyclic voltammetry. The values measured by this technique were found to be in excellent agreement with those obtained independently by ${ }^{13} \mathrm{C}$ NMR spectroscopy.

The basis for the voltammetric determination of $K_{2}$ was the expectation that proton transfer from the oxygen acid, 9-hydroxyanthracene, would be considerably faster than proton transfer from the carbon acid, 9-anthrone. Thus, at a suitable time scale (sweep rate) it would be possible to eliminate only 9-hydroxyanthracene from the diffusion layer by reaction with an electrogenerated base leaving 9-anthrone, the concentration of which could then be determined from the cyclic voltammogram. Obvious prerequisites for this approach are that the interconversion of the two tautomers is negligible at the time scale of the experiment and that the probase is significantly more easily reduced than the 9-hydroxyanthracene/9-anthrone system. 9-Fluorenone was found to fulfil the latter requirement.

The voltammograms resulting from a typical set of experiments are shown in Fig. 1. The reversible couple due to formation of the 9-fluorenone anion radical in DMF in the absence of 9-hydro- 


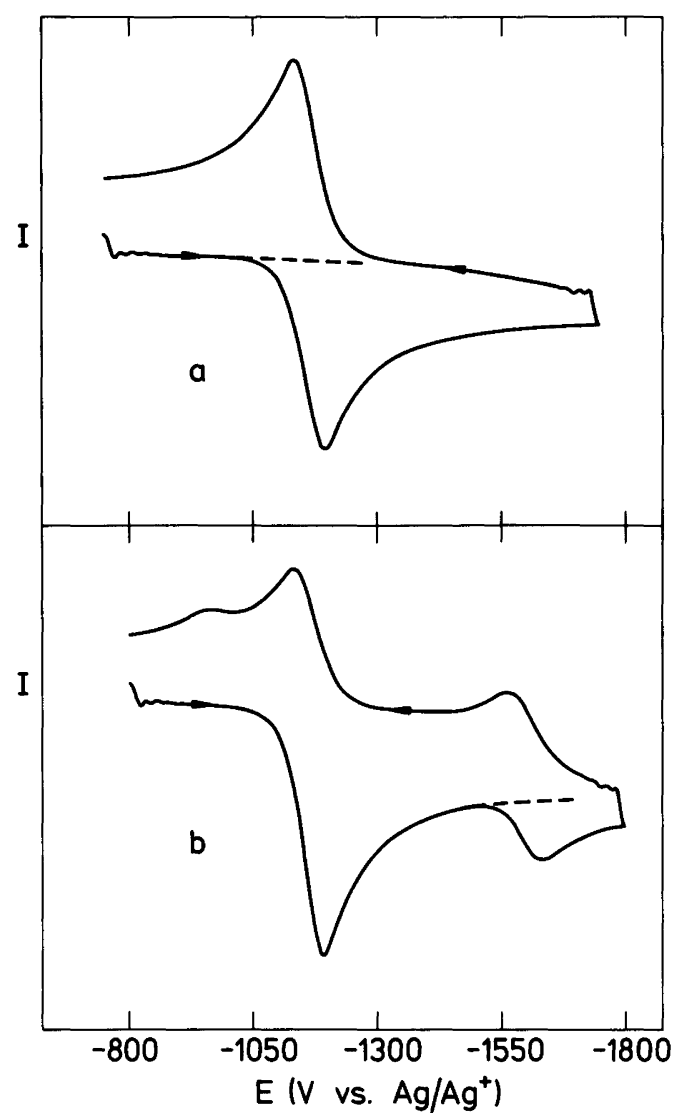

Fig. 1. Cyclic voltammetry at an $\mathrm{Hg}$ working electrode $(d=0.6 \mathrm{~mm})$ of (a) $1 \mathrm{mM}$ 9-fluorenone and (b) $1 \mathrm{mM}$ 9-fluorenone and $1 \mathrm{mM}$ 9-hydroxyanthracene/9anthrone. The solvent was DMF containing $\mathrm{Bu}_{4} \mathrm{NBF}_{4}$ $(0.1 \mathrm{M})$ as supporting electrolyte. $v=100 \mathrm{~V} \cdot \mathrm{s}^{-1}$; $t=22^{\circ} \mathrm{C}$.

xyanthracene/9-anthrone is shown in (a). The concentration was $1 \mathrm{mM}$ and the sweep rate $(v)$ $100 \mathrm{~V} \cdot \mathrm{s}^{-1}$. The voltammogram in (b) is that for the same solution after addition of an equimolar amount of 9-hydroxyanthracene/9-anthrone. It is of interest to notice that the new couple which has appeared at $-1.6 \mathrm{~V} v s . \mathrm{Ag} / \mathrm{Ag}^{+}$is perfectly reversible in contrast to the irreversible couple observed at the same sweep rate for a solution containing only the 9-hydroxyanthracene/9-anthrone system in DMF (not shown). This indicates that 9-hydroxyanthracene, which is the acidic species in the self-protonation reaction, ${ }^{5,6}$ has been effectively consumed by the electrogen- erated base, as expected. To test for the hypothesis that only proton transfer from the hydroxy form is observed at the time scale of the experiment, the concentration of 9-fluorenone was doubled. In DMF the size of the reversible 9-anthrone couple remained essentially unchanged indicating that the concentration of 9-anthrone left in the diffusion layer was not affected by the amount of electrogenerated base. However, in DMSO the reversible 9-anthrone couple was slightly diminished.

It has been shown ${ }^{5,6}$ that moderate structural changes have only a negligible effect on the height of the reversible voltammetric reduction peaks for $1 \mathrm{mM}$ solutions of aromatic ketones related to benzophenone. Representative peak current values at an arbitrary scale are: benzophenone (119), xanthone (118) and fluorenone (121). Accordingly we are allowed to use the peak height for the $1 \mathrm{mM}$ solution of 9-fluorenone as a standard for a hypothetical $1 \mathrm{mM}$ solution of 9-anthrone. The equilibrium constant, $K_{2}$, can then be calculated from eqn. 3, where the peak heights $\left(i_{\mathrm{p}}\right)$ refer to Fig. a (9-fluorenone) and Fig. b (9-anthrone). The values of $i_{\mathrm{p}}$ are measured relative to the extrapolated base lines indicated by the broken lines in the Figure. The values of $K_{2}$ obtained in this manner are given in Table 1.

$$
K_{2}=\frac{i_{\mathrm{p}}(9 \text {-fluorenone })-i_{\mathrm{p}}(9 \text {-anthrone })}{i_{\mathrm{p}}(9 \text {-anthrone })}
$$

The value for DMF was only insignificantly affected by the actual value of $v$ as long as it was kept above $10 \mathrm{~V} \cdot \mathrm{s}^{-1}$, while that for DMSO showed a tendency to increase slightly with de-

Table 1. Values of the equilibrium constant, $K_{2}$, for the tautomeric reaction:

9-anthrone $\rightleftharpoons$ 9-hydroxyanthracene

\begin{tabular}{lll}
\hline Solvent & $K_{2}^{a}$ & $K_{2}^{b}$ \\
\hline DMF & $1.7 \pm 0.2$ & $1.5 \pm 0.1$ \\
DMSO & $3.3 \pm 0.2^{c}$ & $3.4 \pm 0.1$ \\
\hline
\end{tabular}

aDetermined by cyclic voltammetry at $22^{\circ} \mathrm{C}$.

${ }^{b}$ Determined by ${ }^{13} \mathrm{C}$ NMR spectroscopy at $21^{\circ} \mathrm{C}$.

'Maximum value (see text) measured at $v=100$ $\mathrm{V} \cdot \mathrm{s}^{-1}$ and $C_{9-\text { fluorenone }}^{\circ}=1 \mathrm{mM}$. 
creasing sweep rate. This latter observation together with the effect of the probase concentration already described means that the value of $K_{2}$ for DMSO is a maximum value.

Alternatively $K_{2}$ may be determined by ${ }^{13} \mathrm{C}$ NMR spectroscopy. The $22.50 \mathrm{MHz}{ }^{13} \mathrm{C}$ NMR spectra of the anthrone system in DMF and DMSO- $d_{6}$ were superpositions of subspectra due to 9-anthrone and 9-hydroxyanthracene. The large concentration differences among the tautomers found in DMSO- $d_{6}$ solution allowed the direct assignment of signals to the individual tautomers and the chemical shifts were found to be essentially unchanged in going to DMF solution. For calculation of $K_{2}$ only the integrated signals due to the hydrogen bearing carbon atoms were used. These signals were identified by off-resonance decoupling and the spectra were recorded with a pulse delay sufficient to ensure all hydrogen bearing carbon atoms to be completely relaxed between the pulses. The results are likewise given in Table 1 and the agreement between the two techniques is obvious.

It should be emphasized that the voltammetric procedure outlined above rests on the large difference in rates for proton transfer from the two tautomeric forms of 9-anthrone. Since similar differences are to be expected in related cases in which the tautomeric equilibrium features one carbon acid and one oxygen acid, the voltammetric method may prove to be of general value for the determination of the associated equilibrium constants. The range of $K_{2}$-values to which the method is applicable was estimated to be between 0.2 and 5 , the limits being imposed mainly by the necessity to measure accutately the voltammetric peak heights and peak height differences.

Acknowledgement. The electrochemical instrumentation, which has been described, ${ }^{9}$ was purchased through grants from the Carlsberg Foundation and the Danish Natural Science Research Council. O.H. gratefully acknowledges this support.

\section{References}

1. Evans, D. H. In Bard, A. J. and Lund, H., Eds., Encyclopedia of Electrochemistry of the Elements, Vol. 12, Dekker, New York 1978, p. 1.

2. Buchta, R.C. and Evans, D.H. J. Electrochem. Soc. 117 (1970) 1494.

3. Wawzonek, S. J. Electrochem. Soc. 128 (1981) 840.

4. Amatore, C., Capobianco, G., Farnia, G., Sandonà, G., Savéant, J. M., Severin, M. G. and Vianello, E. J. Am. Chem. Soc. 107 (1985) 1815.

5. Almdal, K. The XII. Sandbjerg Meeting on Organic Electrochemistry, June 1985.

6. Almdal, K. and Hammerich, O., manuscript in preparation.

7. Sterk, H. Monatsh. Chem. 100 (1969) 916.

8. Mills, S. G. and Beak, P. J. Org. Chem. 50 (1985) 1216.

9. Nielsen, M. F., Hammerich, O. and Parker, V. D. Acta Chem. Scand. 40 (1986) 101.

Received September 27, 1985. 